\title{
THE FIRST SIGN CHANGE OF A COSINE POLYNOMIAL
}

\author{
JAMES D. NULTON AND KENNETH B. STOLARSKY
}

\begin{abstract}
It is reasonable to expect the first sign change of a real cosine polynomial to decrease when its smallest frequency is increased. Many cases in which this is true are exhibited, but it is shown that there exist (presumably unusual) cosine polynomials for which the first sign change may increase by an arbitrarily large amount.
\end{abstract}

1. Introduction. The position of the first sign change of a real cosine polynomial as a function of its frequencies $\lambda_{i}$ seems unstudied. It is well known, however, that in a small angle about the positive $x$-axis the density of the complex zeros of any

$$
f(x)=\sum_{i=1}^{N} a_{i} \cos \lambda_{i} x
$$

with (say) the $a_{i}$ real and

$$
0<\lambda_{1}<\cdots<\lambda_{N}
$$

is proportional to the largest frequency $\lambda_{N}$ (see, e.g., $[3,4,9,11,12,15]$ for more general results on zero densities of exponential polynomials with arbitrary complex coefficients). For the much harder problem of the real zeros, Kac [5] has shown in certain cases that their density is proportional to a quantity bearing some resemblance to the root mean square of the $\lambda_{i}$.

Hypothesis 1. Increases in the $\lambda_{i}$ tend to decrease the position of the first sign change.

We shall examine several ways of making Hypothesis 1 precise. Our main result is the negative one of $\S 4$; this entails the existence of a curious positive integer $N_{0}$ (see \$2) that may be difficult to compute.

2. Reformulations of Hypothesis 1 . It is obvious that replacing every $\lambda_{i}$ by $\lambda_{i} t$ for some $t>1$ will decrease the position of the first sign change. For $N=1$ Hypothesis 1 is obviously true, and for $N=2$ with $a_{1}=a_{2} \neq 0$ it follows from

$$
\cos \lambda_{1} x+\cos \lambda_{2} x=\frac{1}{2} \cos \left[\left(\frac{\lambda_{1}+\lambda_{2}}{2}\right) x\right] \cos \left[\left(\frac{\lambda_{1}-\lambda_{2}}{2}\right) x\right] \text {. }
$$

On the other hand, for $\lambda>1$ it is not always true for

$$
\cos x+a \cos \lambda x
$$

Received by the editors December 12, 1980 and, in revised form, January 31, 1981.

1980 Mathematics Subject Classification. Primary 33A10, 42 A05.

Key words and phrases. Cosine polynomial, exponential polynomial, frequencies, sign changes, zeros. 
for example, when $a=.1$ the first sign change is $\pi / 2$ when $\lambda=1$, but somewhat less when $\lambda=2$. However, it is not hard to establish

Proposition 2.1. If $0<\lambda_{1}<\lambda_{2}$ and $a$ is real, the first sign change of

$$
\cos \lambda_{1} x+a \cos \lambda_{2} x
$$

will not increase if $\lambda_{1}$ is replaced by $\lambda^{*}$, where

$$
\lambda_{1} \leqslant \lambda^{*} \leqslant \lambda_{2}
$$

Here, and in what follows, we omit proofs of statements that can be established in a fairly straightforward manner involving implicit differentiation.

The above result suggests

Hypothesis 2. Increases in the LOWEST frequency tend to decrease the position of the first sign change.

That nothing much stronger than this can be invariably true, even when all the $a_{i}$ are equal, is indicated by the fact that the first sign change of

$$
\cos \lambda_{1} x+\cos \lambda_{2} x+\cos \lambda_{3} x, \quad 0<\lambda_{1}<\lambda_{2}<\lambda_{3},
$$

is increasing with $\lambda_{2}$ for

$$
7 \leqslant \lambda_{2} \leqslant 7.3
$$

when $\lambda_{1}=1$ and $\lambda_{3}=16$ (whether Hypothesis 2 is valid for all trinomials (2.5) remains open). The result of $\S 4$ is that Hypothesis 2 is sometimes false. It would be interesting to know the smallest integer $N=N_{0}$ for which it fails; clearly $N_{0}>3$.

It is not hard to establish the following weakened form of Hypothesis 1 .

Proposition 2.2. Let

$$
P=\left(\lambda_{01}, \ldots, \lambda_{0 N}\right)
$$

be a point in $E^{N}$. Assume that for all $\left(\lambda_{1}, \ldots, \lambda_{N}\right)$ in the neighborhood of $P$ the first sign change of the cosine polynomial (1.1) is a continuous function of the $\lambda_{i}$. Then for some $i$ it is locally a decreasing function of $\lambda_{i}$ when the $\lambda_{j}$ with $j \neq i$ are held fixed.

3. Reformulations of Hypothesis 2. The following results assert the validity of Hypothesis 2 for certain lacunary polynomials, and for polynomials with a dominant first term. Their rather simple nature is brought out by stating them for a class of functions wider than cosine polynomials; proofs are omitted.

Proposition 3.1. Let $g(x)$ be an even real-valued function of period $2 \pi$ that is continuously differentiable, and strictly decreasing on $[0, \pi)$. Assume that

$$
g(0)=1, \quad g(\pi / 2)=0 .
$$

Let

$$
G(x)=g\left(\lambda_{1} x\right)+a_{2} g\left(\lambda_{2} x+c_{2}\right)+\cdots+a_{n} g\left(\lambda_{N} x+c_{N}\right)
$$

where the $a_{i}, c_{i}$, and $\lambda_{i}$ are real numbers such that

$$
1 \leqslant \lambda_{1}<\lambda_{2}<\cdots<\lambda_{N}
$$

Assume that

$$
4 \lambda_{j} \leqslant \lambda_{j+1}, \quad 1 \leqslant j \leqslant N-1 .
$$


Then whenever the first sign change of $G(x)$ is a continuous function of $\lambda_{1}$, and

$$
1<\lambda_{1}<\lambda_{2} / 4 \text {, }
$$

that sign change is a nonincreasing function of $\lambda_{1}$.

Proposition 3.2. If condition (3.1) is augmented to

$$
g(0)=1, \quad g(\pi / 2)=0, \quad g(\pi)=-1
$$

and condition (3.4) is replaced by

$$
\max _{0<x<2 \pi}|g(x)| \sum_{i=2}^{N}\left|a_{i}\right|<1,
$$

then the conclusion of Proposition 3.1 is valid for

$$
1 \leqslant \lambda_{1} \leqslant \lambda_{2} \text {. }
$$

4. Hypothesis 2 is sometimes false: The proof of the negative result below is similar in spirit to some work of Talalyan ([17], esp. pp. 113-114; see also [13]), although his goals are rather different.

ThEOREM. Let $m \geqslant 2$ be an integer and $0<\varepsilon<(6 m)^{-1}$. There is a real cosine polynomial

$$
T\left(x ; \lambda_{1}\right)=T\left(x ; \lambda_{1}, \ldots, \lambda_{N}\right)=\sum_{j=1}^{N} a_{j} \cos \lambda_{j} x, \quad 1<\lambda_{1}<\cdots<\lambda_{N},
$$

such that the first sign change of $T(x ; 1+\varepsilon)$ exceeds the first sign change of $T(x ; 1)$ by more than $m$.

It is easy to see that the theorem would be false without some upper bound on $\varepsilon$; we leave open the question of how much our upper bound may be relaxed. The expressions $\varepsilon, g(x), m, \mu$, and $y_{0}$ shall have the same meaning throughout this section. The theorem follows from Lemma 3 upon replacing $x$ by $x / m$ and $m_{1}$ by $m$ in (4.12).

LEMMA 1. Let $x>0$ and

$$
g(x)=\cos m x-\cos (1+\varepsilon) m x=2 \sin (1+\varepsilon / 2) m x \sin (\varepsilon m x / 2) .
$$

Then $|g(x)|<x / 6$ and

$$
g(x)>0, \quad 0<x<3 \pi / 8 m .
$$

Also, there is a number $\mu$ with $0<\mu<\pi / 16 m$ such that

$$
\mu<g(x), \quad \pi / 8 m<x<3 \pi / 8 m \text {. }
$$

Proof. This is almost trivial; note that in the above intervals $g(x)$ is strictly increasing, so we may take

$$
\mu=g(\pi / 8 m)<\pi / 16 m .
$$

LEMMA 2. For $x \geqslant 0$ define

$$
h(x, y)=x+y \cos 4 m x, \quad v(y)=\min _{x} h(x, y) .
$$

There is a positive number $y_{0}<5 \pi / 16 \mathrm{~m}$ such that

$$
v\left(y_{0}\right)=-\mu
$$




$$
h\left(x, y_{0}\right)<0 \text { implies } \pi / 8 m<x<3 \pi / 8 m \text { or } x=0 \text {, }
$$

and

$$
h\left(x, y_{0}\right)+g(x)>0 .
$$

Proof. The function $v(y)$ assumes values arbitrarily close to 0 for small positive $y$, while

$$
v(5 \pi / 16 m) \leqslant h(\pi / 4 m, 5 \pi / 16 m)=-\pi / 16 m .
$$

Since $v$ is continuous, (4.7) follows. Clearly $h\left(x, y_{0}\right)$ is positive to the left of the interval of (4.8); to the right it is at least $3 \pi / 8 m+5 \pi / 16 m(-1)>0$.

Inequality (4.9) is clear for $0 \leqslant x \leqslant \pi / 8 m$, and by (4.4) and (4.7) it extends to $x<3 \pi / 8 m$. Beyond this range the left side exceeds

$$
x-y_{0}-x / 6 \geqslant 5 x / 6-5 \pi / 16 m>0 .
$$

LEMMA 3. There is a real cosine polynomial

$$
p\left(x ; m_{1}\right)=-\cos m_{1} x+\sum_{j=m+1}^{J} b_{j} \cos j x
$$

such that the first sign change of $p(x ;(1+\varepsilon) m)$ exceeds the first sign change of $p(x ; m)$ by more than 1 .

Proof. Standard arguments from Fourier analysis enable us to extend

$$
F(x)=x+\cos m x+.5 \mu, \quad 0<x<2,
$$

to an even continuous function $f$, periodic on $[-\pi, \pi]$, for which

$$
\int_{-\pi}^{\pi} f(x) \cos j x d x=0, \quad 0<j<m .
$$

The Cesàro means of its Fourier series converge to $f$ uniformly, so there is a real cosine polynomial $C(x)$ with all frequencies greater than $m$ such that

$$
x<C(x)-\cos m x<x+\mu, \quad 0<x<2 .
$$

Define

$$
k(x)=C(x)-\cos m x+y_{0} \cos 4 m x
$$

then

$$
h\left(x, y_{0}\right)<k(x)<h\left(x, y_{0}\right)+\mu, \quad 0<x<2 .
$$

By (4.7) and (4.8), $k(x)$ has a sign change at some $x_{0}<3 \pi / 8 m<1$. However, to replace $\cos m x$ by $\cos (1+\varepsilon) m x$ is to add $g(x)$ to $k(x)$, and by (4.17) and (4.9) this yields a cosine polynomial that is strictly positive on $[0,2]$. This completes the proof.

It is plausible that in $\left(\lambda, \ldots, \lambda_{N}, a_{1}, \ldots, a_{N}\right)$ phase space, the set of points where Hypothesis 2 fails in asymptotically very small in relative measure.

5. Remarks. Problems concerning the size (e.g. $L_{p}$-norm) and real zero distribution of phase-shifted cosine polynomials $f(x)=\sum_{i-1}^{N} a_{i} \cos \left(\lambda_{1} x+c_{i}\right)$ or even pure cosine polynomials (i.e. every $c_{i}$ is zero) are often difficult $[6$, p. 33; 8]; the 
establishment of some simply stated results (even when every $a_{i}$ is one) has required great ingenuity $[1,2,7,8,14,18]$. Indeed, simple questions can already require extensive analysis for $N=3$ [16].

\section{REFERENCES}

1. P. J. Cohen, On a conjecture of Littlewood and idempotent measures, Amer. J. Math. 82 (1960), 191-212.

2. H. Davenport, On a theorem of P. J. Cohen, Mathematika 7 (1960), 93-97.

3. D. G. Dickson, The asymptotic distribution of zeros of exponential sums, Publ. Math. Debrecen 11 (1964), 295-300.

4. Z_ Zeros of exponential sums, Proc. Amer. Math. Soc. 78 (1965), 84-89.

5. M. Kac, On the distribution of values of trigonometric sums with linearly independent frequencies, Amer. J. Math. 65 (1943), 609-615.

6. J. E. Littlewood, Some problems in real and complex analysis, Heath Math. Mono., Heath, Lexington, Mass., 1968.

7. S. K. Pichorides, A lower bound for the $L^{1}$ norm of exponential sums, Mathematika 21 (1974), 155-159.

8. __ Norms of exponential sums, Publ. Math. D’Orsay, nos. 77-73, Université de Paris-Sud, Orsay, 1976.

9. G. Polya, Geometrisches über die Verteilung der Nullstellen gewisser ganzer transzendenter Funktionen, Münchener Sitzungsberichte 50 (1920), 285-290.

10. On polar singularities of power series, and of Dirichlet series, Proc. London Math. Soc. (2) 33 (1932), 85-101 (esp. p. 89).

11. A. J. van der Poorten, On the number of zeros of functions, Enseign. Math. 23 (1977), 19-38.

12. A. J. van der Poorten and R. Tijdeman, On common zeros of exponential polynomials, Enseign. Math. 21 (1975), 57-67.

13. J. J. Price, Topics in orthogonal functions, Amer. Math. Monthly 82 (1975), 594-609.

14. K. F. Roth, On casine polynomials corresponding to sets of integers, Acta Arith. 24 (1973), 87-98.

15. E. Schwengeler, Geometrisches über die Verteilung der Nullstellen spezieller ganzer funktionen, Thesis, Zürich, 1925.

16. P. Stein, On the real zeros of a certain trigonometric function, Proc. Cambridge Philos. Soc. 31 (1935), 455-457.

17. A. A. Talalyan, The representation of measurable functions by series, Russian Math. Surveys 15 (5) (1960), 77-136.

18. M. Uchiyama (née Katayama) and S. Uchiyama, On the casine problem, Proc. Japan Acad. 36 (1960), 475-479.

Department of Mathematics, University of Illinois at Urbana-Champaign, Urbana, Illinois 61801 (Current address of K. B. Stolarsky)

Current address (J. D. Nulton): Department of Mathematics, San Diego City College, 1313 Twelfth Avenue, San Diego, California 92101 